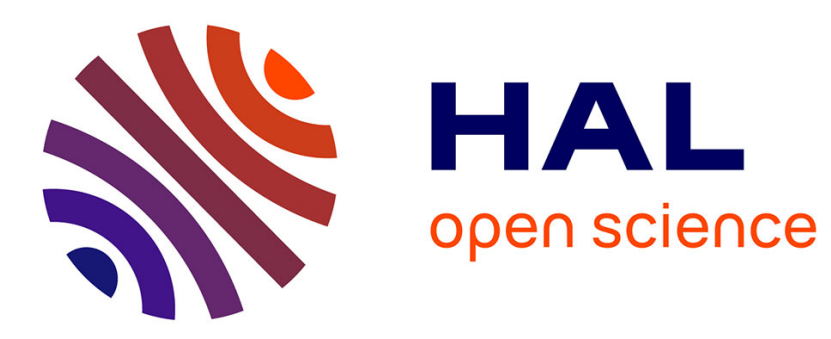

\title{
Symbolic Computation with Symmetric Polynomials an Extension to Macsyma
}

\author{
Annick Valibouze
}

\section{To cite this version:}

Annick Valibouze. Symbolic Computation with Symmetric Polynomials an Extension to Macsyma. Erich Kaltofen and Stephen M. Watt. Computers and Mathematics, Springer-Verlag NY, pp.308320, 1989, 978-0-387-97019-6 10.1007/978-1-4613-9647-5_35 . hal-01672106

\section{HAL Id: hal-01672106 https://hal.sorbonne-universite.fr/hal-01672106}

Submitted on 23 Dec 2017

HAL is a multi-disciplinary open access archive for the deposit and dissemination of scientific research documents, whether they are published or not. The documents may come from teaching and research institutions in France or abroad, or from public or private research centers.
L'archive ouverte pluridisciplinaire HAL, est destinée au dépôt et à la diffusion de documents scientifiques de niveau recherche, publiés ou non, émanant des établissements d'enseignement et de recherche français ou étrangers, des laboratoires publics ou privés. 


\title{
SYMBOLIC COMPUTATION WITH SYMMETRIC POLYNOMIALS AN EXTENSION TO MACSYMA
}

\author{
Annick Valibouze \\ LITP, \\ 4, Place Jussieu, 75252 Paris Cedex 05 \\ and "GRECO DE CALCUL FORMEL" No 60 \\ UUCP:avb@litp.univ-p6-7.fr
}

\begin{abstract}
This paper presents SYM, an extension package to MACSYMA symbolic manipulation system. SYM allows to deal efficiently with symetric polynomials which appear to be useful in several symbolic algorithms such as the computation of resolvants, resultants or minimal polynomials.

The SYM package proposes various algorithms which allows to test the symetricity of polynomials, to compute orbits of symetric polynomials, to make algebraic operations on them, to realize change of basis for the symetric polynomials algebra and so on. Our algorithms are very efficient because they use a particular representation of the symetric polynomials using only one monomial associated with each orbit. This contracted representation has been possible thanks to the symetricity of the polynomials that we consider. The main resulting property is that we avoid the combinatory explosion associated to the exponential development of the symetric group of the variables of a polynomial.

Like MACSYMA, the SYM extension is written in the Lisp programming language because it uses heavily MACSYMA primitive tools. SYM has been developped under UNIX 4.3 bsd in Franzlisp.

Our presentation will include a description of the SYM package and some interesting applications built on SYM. The paper will be an english version of the joined presentation of the system.
\end{abstract}

\section{INTRODUCTION}

We present here a package of manipulations of symmetric polynomials implemented in Franzlisp. This package, called SYM, constitutes at present an extension of the system of symbolic computation MACSYMA. It performs a few manipulations on symmetric polynomials; it can also be used for direct applications. Some algorithms extend easily to functions that are symmetric with respect to sets of variables (i.e. multi-symmetric functions); these functions will be dealt with in the present paper. 


\section{Definitions and notations}

\subsection{Partitions and multi-partitions}

Let us first introduce the notion of a partition, which is the basic object that allows us to represent the symmetric polynomials in the most possible contracted form. For more details, the reader is referred to [Andrews] or [Macdonald].

A partition is a finite or infinite sequence $I=\left(i_{1}, i_{2}, \ldots, i_{n}, \ldots\right)$ of non-negative integers in decreasing order: $i_{1} \geq i_{2} \geq \cdots i_{n} \geq \cdots$, and containing only a finite number of non-zero terms. We make the convention that sequences only differing by the number of zeros at the end are equal. For example $(2,1)$ and $(2,1,0,0)$ are the same partition. The non-zero $i_{k}$ of $I$ are called the parts of $I$. The number of parts is the length of $I$ and the sum of the parts is the weight of $I$. We shall call multi-partition of order $p$ a finite sequence $I$ of length $p, I=\left(I_{1}, I_{2}, \ldots, I_{p}\right)$, where each $I_{k}$ is a partition.

\subsection{Generalities about symmetric functions}

Let $\mathcal{A}$ be a ring, and let $D=\left(d_{1}, d_{2}, \ldots, d_{p}\right)$ be an element of $\mathbf{N}^{p}$ with $d_{1}+d_{2}+\cdots+d_{p}=n$. Let $X=\left(x^{(1)}, x^{(2)}, \ldots, x^{(p)}\right)$, where each $x^{(r)}$ is an alphabet of $d_{r}$ variables $x_{1}^{(r)}, x_{2}^{(r)}, \ldots, x_{d_{r}}^{(r)}$. Then $R_{D}=\mathcal{A}[X]$ is the ring of polynomials in the $n$ variables $x_{i}^{(r)}\left(i=1, \ldots, d_{r}\right.$ and $\left.r=1, \ldots, p\right)$ with coefficients in $\mathcal{A}$. The product $S_{d_{1}} \times S_{d_{2}} \times \cdots \times S_{d_{p}}$ of the symmetric groups $S_{d_{i}}$ will be denoted by $S_{D}$.

For each element $\sigma$ of $S_{n}$ and each finite sequence of $n$ elements $T=\left(t_{1}, t_{2}, \ldots, t_{n}\right), \sigma(T)$ is the sequence $\left(t_{\sigma(1)}, t_{\sigma(2)} \ldots t_{\sigma(n)}\right)$. This generalizes as follows: let $T=\left(t^{(1)}, t^{(2)}, \ldots, t^{(p)}\right)$ be a $p$-tuple of finite sequences $t^{(r)}$ of $d_{r}$ element. For each element $\sigma=\left(\sigma_{1}, \sigma_{2}, \ldots, \sigma_{p}\right)$ of $S_{D}$ we define:

$$
\sigma(T)=\left(\sigma_{1}\left(t^{(1)}\right), \sigma_{2}\left(t^{(2)}\right), \ldots, \sigma_{p}\left(t^{(p)}\right)\right) .
$$

$G_{S_{D}}(T)$ will be the stabilizer of $T$ under the action of $S_{D}$ (the subgroup of the elements of $S_{D}$ leaving $T$ unchanged). If $f \in R_{D}, O_{S_{D}}(f)$ will denote the orbit of $f$ under $S_{D}$, i.e., the set of polynomials $h$ of $R_{D}$ such that $h(X)=f(\sigma(X))$ for an element $\sigma$ of $S_{D}$.

A polynomial $P$ of $R_{D}$ is said to be multi-symmetric of order $D$ if $P(X)=P(\sigma(X))$ for all $\sigma \in S_{D}$ (i.e. $\left.\operatorname{card}\left(O_{S_{D}}(P)\right)=1\right)$. This algebra of invariants will be denoted by $R_{D}^{S_{D}}$. If $p=1$ we simply say that $P$ is symmetric .

For $p=1$ we take $D=d_{1}=n$ and $X=\left(x_{1}, x_{2}, \ldots, x_{n}\right)$. If $U=\left(u_{1}, u_{2}, \ldots, u_{n}\right)$ is an element of $\mathbf{N}^{n}$, we define the monomial $X^{U}$ by:

$$
X^{U}=x_{1}^{u_{1}} x_{2}^{u_{2}} \ldots x_{n}^{u_{n}},
$$

and if $U$ is a $D$-tuple having $p$ finite sequences of integers $u^{(1)}, \ldots, u^{(p)}$ of length $d_{1}, \ldots, d_{p}$, respectively, then:

$$
X^{U}=\left(x^{(1)}\right)^{u^{(1)}} \ldots\left(x^{(p)}\right)^{u^{(p)}} .
$$

With a multi-partition $I$ we associate the monomial form $M_{I}(X)$, which is the sum of the elements of the orbit of $X^{I}$ under the $S_{D^{-a c t i o n}}$ :

$$
M_{I}(X)=\sum_{\sigma \in S_{D} / G_{S_{D}}(I)} X^{\sigma(I)} .
$$


Examples:

- $p=1-M_{(3,2,2)}(x, y, z)=x^{3} y^{2} z^{2}+y^{3} x^{2} z^{2}+z^{3} x^{2} y^{2}$.

- $p=2$ - For $X=((x, y),(a, b, c))$ we have $M_{((3,2),(1,1))}(X)=x^{3} y^{2} a b+x^{3} y^{2} a c+x^{3} y^{2} b c+y^{3} x^{2} a b+$ $y^{3} x^{2} a c+y^{3} x^{2} b c$.

\subsection{Contracted and partitioned forms}

A monomial form $M_{I}(X)$ will be represented either by a monomial $X^{J}$ of the orbit of $X^{I}$, called a contracted monomial form, or by the partition $I$. If we give it a coefficient, then the monomial form is represented by a contracted term or by a partitioned term, the latter being a list in which the first element is the coefficient and the rest is the partition. We can now represent a symmetric polynomial (or a multi-symmetric polynomial) by a contracted polynomial, the sum of the contracted terms, or by a partitioned polynomial, the list of the partitioned terms.

For example the contracted polynomial associated with the polynomial $3 x^{4}+3 y^{4}-2 x y^{5}-2 x^{5} y$, symmetric in the variables $x$ et $y$, is $3 x^{4}-2 x y^{5}$ and the partitioned polynomial is $[[3,4],[-2,5,1]]$.

\subsection{Types of arguments}

For the description of the function we use the following notations:

card is the cardinality of the set of the variables.

$e_{i}: i^{\text {th }}$ elementary symmetric function

$p_{i}: i^{\text {th }}$ power function

$1_{-} \mathrm{ele}=\left[e_{1}, e_{2}, e_{3}, \ldots, e_{n}\right]$, where the number $n$ is important in some definitions of the function

$1_{-} \mathrm{cele}=\left[\mathrm{card}, e_{1}, e_{2}, e_{3}, \ldots, e_{n}\right]$

$1_{-}$pui $=\left[p_{1}, p_{2}, p_{3}, \ldots, p_{m}\right]$

$1_{-}$cpui $=\left[\right.$card $\left., p_{1}, p_{2}, p_{3}, \ldots, p_{m}\right]$

sym $<--->$ is a symmetric polynomial, but the representation is not specified

fmc $<--->$ contracted monomial form

part $<--->$ partition

tc $<--->$ contracted term

tpart $<--->$ partitioned term

psym $<--->$ symmetric polynomial in its extended form

pc $<--->$ symmetric polynomial in a contracted form

multi_pc $<--->$ multi-symmetric polynomial in a contracted form under $S_{D}$

ppart $<--->$ symmetric polynomial in its partitioned form

$\mathrm{P}\left(x_{1}, \ldots, x_{q}\right)$ is a polynomial in the variables $x_{1}, \ldots, x_{q}$

lvar is a list of variables of $X$ in the case $p=1$. $\left[\operatorname{lvar}_{1}, \ldots, \operatorname{lvar}_{p}\right]$ is a list of lists of variables representing the multi-alphabet $X$ and where the variables of $\operatorname{lvar}_{j}$ represent the $d_{j}$ variables of the alphabet $x^{(j)}$.

\section{Remarks :}

1- The functions of SYM can complete the lists, such as $1_{-}$cele, with formal values. This values are ei for the $i^{\text {th }}$ elementary symmetric function and pi for the $i^{\text {th }}$ power function.

2- There exist many kinds of evaluations for the polynomials under MACSYMA : ev, expand, rat, ratsimp. With SYM the choice is possible with a flag oper. In each call of a function, SYM tests if the variable oper is modified. In this case, the modification is made as follows: if oper = meval, it uses the ev mode, if oper = expand, it uses the expand mode (it is more efficient 
for the numeric calculations), if oper $=$ rat, it uses the rat form and if oper $=$ ratsimp, it uses the ratsimp form.

\section{Description of the available functions}

\subsection{Change of representation}

- tpartpol (psym,lvar) $\longrightarrow$ ppart , partpol (psym, lvar) $\longrightarrow$ ppart give, in the lexicographic order, increasing and decreasing, respectively, the partioned polynomial associated with the polynomial psym. The function tpartpol tests if the polynomial psym is actually symmetric.

- $\operatorname{contract(psym,lvar)~} \longrightarrow$ pc tcontract (psym, lvar) $\longrightarrow \mathrm{pc}$ act as partpol and give the contracted form.

- cont2part (pc,lvar) $\longrightarrow$ ppart gives the partitioned polynomial associated with the contracted form pc.

- part2cont (ppart, Ivar) $\longrightarrow$ pc gives a contracted form associated with the partioned form ppart.

- explose(pc,lvar) $\longrightarrow$ psym gives a contracted form associated with the extended form psym. 
Examples:

$$
\begin{gathered}
\operatorname{tpartpol}\left(\operatorname{expand}\left(\mathrm{x}^{\wedge} 4+\mathrm{y}^{\wedge} 4+\mathrm{z}^{\wedge} 4-2 *(\mathrm{x} * \mathrm{y}+\mathrm{x} * \mathrm{z}+\mathrm{y} * \mathrm{z})\right),[\mathrm{x}, \mathrm{y}, \mathrm{z}]\right) ; \\
{[[1,4],[-2,1,1]]}
\end{gathered}
$$

Now suppose that the polynomial $2 a^{3} * b * x^{4} * y$ is the contracted form of a symmetric polynomial in $\mathbf{Z}[x, y, z]$.

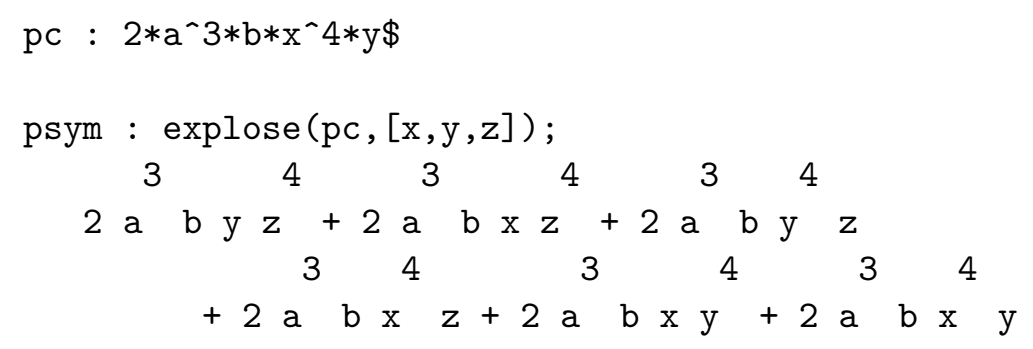

If we use the function contract we find again the contracted form:

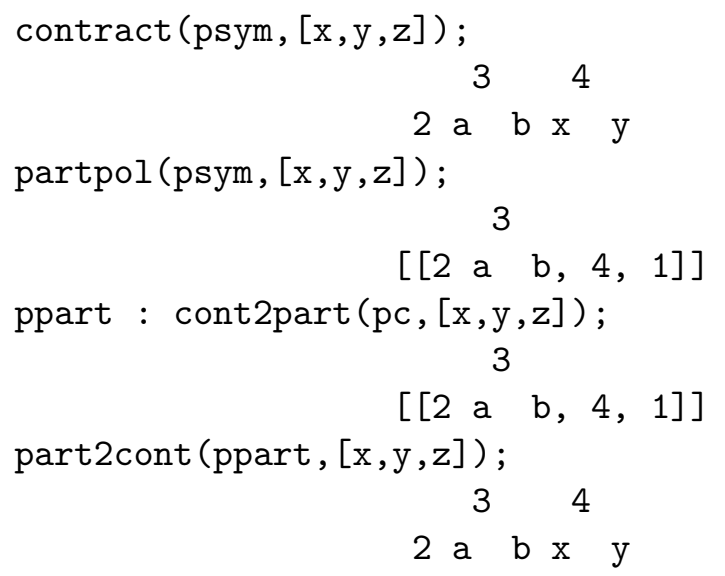

\subsection{The partitions}

- kostka (part1, part2) (written by P.ESPERET) gives the Kostka number associated with the partitions part1 et part2.

- treinat (part) $\longrightarrow$ list of the partitions that are less than the the partition part in the natural order and that are of the same weight.

- $\operatorname{treillis}(\mathrm{n}) \longrightarrow$ list of the partitions of weight equal to $n$.

- lgtreillis $(\mathrm{n}, \mathrm{m})$

- lgtreillis $(\mathrm{n}, \mathrm{m}) \longrightarrow$ list of the partitions of weight equal to $n$ and of length equal to $m$.

- Itreillis $(\mathrm{n}, \mathrm{m})$

- lgtreillis $(\mathrm{n}, \mathrm{m}) \longrightarrow$ list of the partitions of weight equal to $n$ and the length less than or equal to $m$. 


\subsection{The orbits}

- $\operatorname{orbit}\left(\mathrm{p}\left(x_{1}, \ldots, x_{n}\right)\right.$, lvar $) \longrightarrow O_{S_{n}}(\mathrm{p})$ gives the list of polynomials of the orbit $O_{S_{n}}(\mathrm{p})$ where the $n$ variables of $\mathrm{p}$ are in the list lvar. This function does not consider the possible symmetries of $\mathrm{p}$.

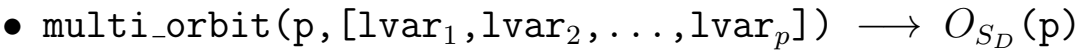
gives the orbit of $\mathrm{p}$ under $S_{D}$ (see above), where the variables of the multi-alphabet $X$ are in the lists $1 \operatorname{var}_{i}$.

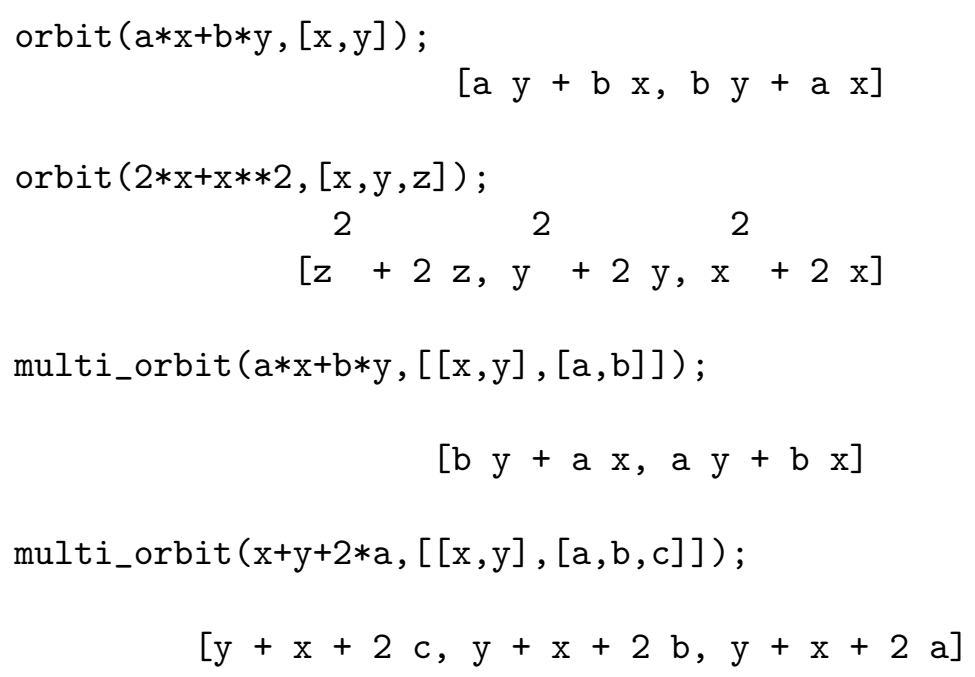

\subsection{Contracted product of two symmetric polynomials}

The formula is in [V1] or [V2] and the proof in [V2].

- multsym(ppart1, ppart2,card) $\longrightarrow$ ppart The arguments are two partioned forms and the cardinality. The result is in partitioned form.

For example, take two symmetric polynomials in their contracted form. We first compute the product in the standard way and then with the function multsym. We are in $\mathbf{Z}[x, y]$ and the two contracted forms pc1 and pc2 are associated with the two symmetric polynomials p1 and p2.

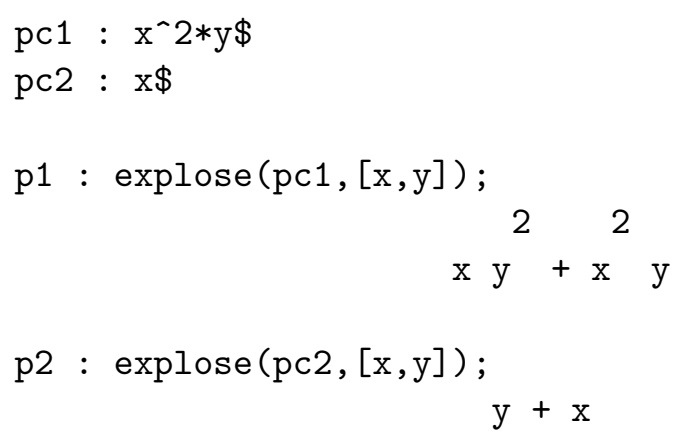

There is the product of the two symmetric polynomials with the standard operation of MACSYMA:

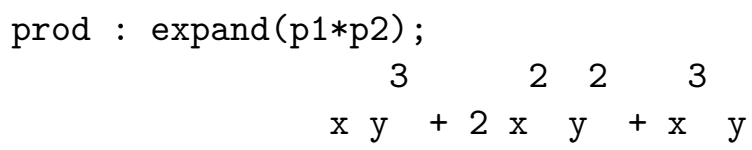

we verify below that this is the extended form of the product obtained with the function multsym: 


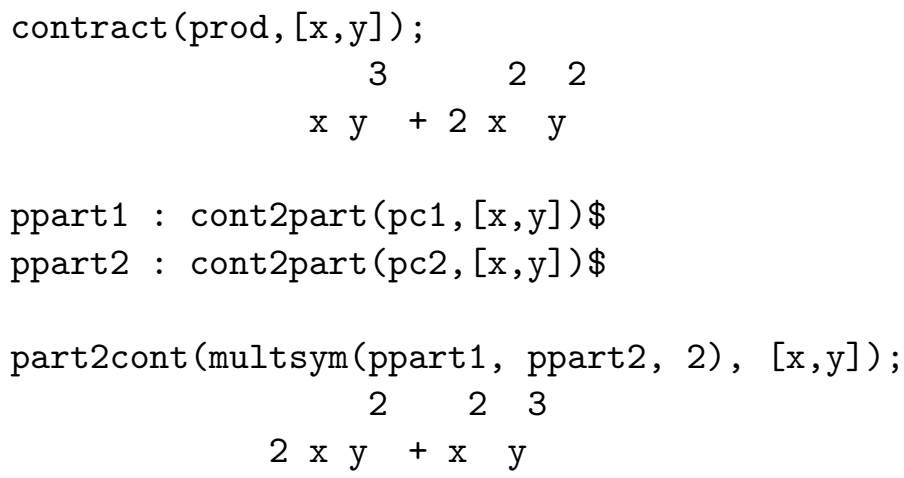

\subsection{Change of basis}

The monomial forms $M_{I}(X)$ where $I$ varies in the set of partitions of length $\leq n$ are an $\mathcal{A}$-base of the free $\mathcal{A}$-module $R_{D}^{S_{D}}$. The Schur functions also form an $\mathcal{A}$-base of the free $\mathcal{A}$-module. We have the following algebra bases: the elementary symmetric functions $(\mathcal{A}$-base), the power functions ( $\mathbf{Q}$-base if $\mathcal{A}=\mathbf{Z}$ ) and the complete functions ( $\mathcal{A}$-base).

When $I=(\overbrace{1,1, \ldots, 1}^{i}, 0,0, \ldots, 0)$ where $0 \leq i \leq n$, the monomial form $e_{i}(X)=M_{I}(X)$ is also called the $i^{\text {th }}$ elementary symmetric function over $X$, with the convention $e_{0}=1$ and $e_{i}=0$ for $i>n$. When $I=(i)$, the monomial form $p_{i}(X)=M_{I}(X)=\sum_{x \in X} x^{i}$ is called the $i^{t h}$ power function over $X,\left(p_{0}=n\right)$. The $i^{\text {th }}$ complete symmetric function, $h_{i}(X)$, is the sum of the monomial forms $M_{I}(X)$ where the weight of $I$ is $i,\left(h_{0}=1\right.$ and $h_{r}=0$ if $\left.r<0\right)$.

Let $\mathcal{M}$ be a matrix and $I=\left(i_{1}, i_{2}, \ldots, i_{n}\right)$ a sequence of $\mathbf{Z}^{n}$; let $\mathcal{M}_{I}$ be the minor of $\mathcal{M}$ constructed with the lines $1,2, \ldots, n$ and the columms $i_{1}+1, i_{2}+2, \ldots, i_{n}+n$, with the convention $\mathcal{M}_{I}=0$ if there exists $r$ such that $i_{r}+r \leq 0$.

Let $S=\left(h_{i-j}\right)_{i \geq 1, j \geq 1}$ be an infinite matrix:

$$
\left(\begin{array}{ccccc}
h_{0} & h_{1} & h_{2} & h_{3} & \cdots \\
0 & h_{0} & h_{1} & h_{2} & \cdots \\
0 & 0 & h_{0} & h_{1} & \cdots \\
0 & 0 & 0 & h_{0} & \cdots \\
0 & 0 & 0 & 0 & \cdots \\
\vdots & \vdots & \vdots & \vdots & \vdots
\end{array}\right)
$$

where the $h_{i}$ are the complete functions. We call Schur function of index I the minor $S_{I}$. 
- $\operatorname{elem}\left(1_{-}\right.$cele, sym,lvar $) \longrightarrow P(e 1, \ldots$, eq $)$

decomposes the symmetric polynomial sym into the elementary symmetric functions with the algorithm in [V1].

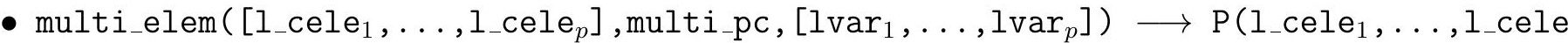
We have the multi-symmetric polynomial multi_pc in its contracted form. This function decomposes successively in each package $\mathbf{l}_{-} c e l e_{j}$ of elementary symmetric functions of the alphabet $x^{(j)}$ (see the section 1.2).

- $\operatorname{pui}\left(1_{-}\right.$cpui, sym, Ivar $) \longrightarrow \mathrm{P}(\mathrm{p} 1, \ldots, \mathrm{pq})$ decomposes the symmetric polynomial sym into the power functions with the algorithm in [V1].

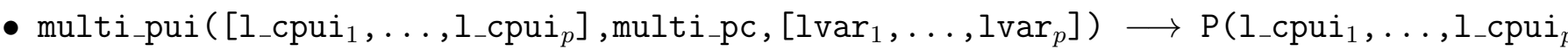
see multi_elem.

In this examples the symmetric polynomials are in contracted form.

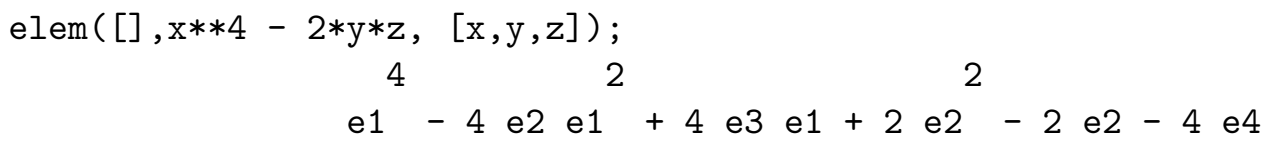

If the cardinality is 3 we have:

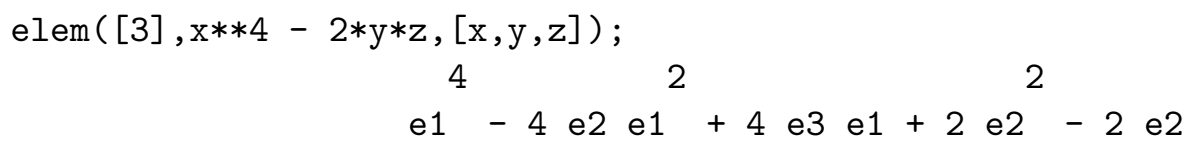

If the cardinality is 3 and $e_{1}=7$ we have:

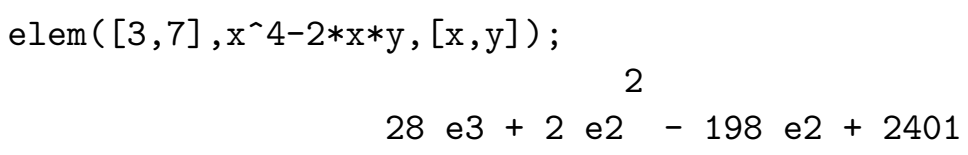

For the power functions we know that if the cardinality of the alphabet $X$ is $n$ then the $i^{t h}$ power function, $i>n$, depends algebraically on the $p_{1}, p_{2}, \ldots, p_{n}$. For this reason, when the function pui completes the list $1_{-}$cpui with formal values and the degree of the polynomial sym is greater than $n$, the $i^{\text {th }}$ power functions for $i>n$ do not appear. For this computation the function pui uses the function puireduc (see below).

For following formulas for the change basis we refer to [Macdonald] and [Lascoux, Schützenberger].

- ele2pui(m,1_cele) $\longrightarrow$ 1_cpui gives the first $\mathrm{m}$ power functions as functions of the elementary symmetric functions with the [Girard]-Newton formulas.

- $\operatorname{pui} 2 \mathrm{ele}\left(\mathrm{n}, 1_{-} \mathrm{cpui}\right) \longrightarrow 1_{-} \mathrm{cele}$ gives the elementary symmetric functions when we know the power functions. If the flag pui2ele is girard, the result is the first $n$ elementary symmetric functions and if its is close, the result is the $n^{\text {th }}$ elementary symmetric function.

In the following example we find the first 3 elementary symmetric functions when the power functions are generic. 
$\operatorname{pui} 2 \mathrm{ele}(3,[])$;

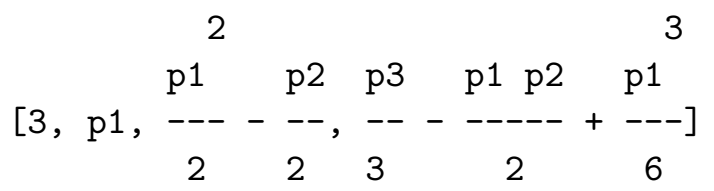

Now the cardinality of the alphabet $X$ is 3 and the first power function is equal to 2 . We remark that the $4^{\text {th }}$ elementary symmetric function is zero, because the cardinality is 3 . We compute the first three power functions below.

$\operatorname{pui} 2 \mathrm{ele}(4,[3,2])$;

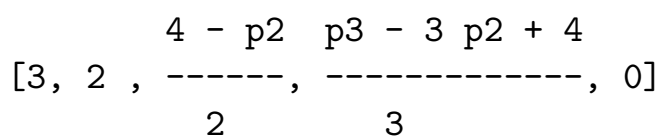

ele2pui (3, []);

$$
\left[3, \mathrm{e} 1, \mathrm{e}^{2}-2 \mathrm{e} 2,3 \mathrm{e} 3-3 \mathrm{e} 1 \mathrm{e} 2+\mathrm{e} 1^{3}\right]
$$

In the next example, since the cardinality is 2 , the $3^{\text {th }}$ elementary symmetric function is zero: ele2pui (3, [2]);

$$
\left[2, \mathrm{e} 1, \mathrm{e}^{2}-2 \mathrm{e} 2, \mathrm{e} 1^{3}-3 \text { e1 e2 }\right]
$$

- $\operatorname{puireduc}\left(\mathrm{n}, \mathrm{I}_{-} \mathrm{cpui}\right) \longrightarrow\left[\mathrm{card}, p_{1}, p_{2}, p_{3}, \ldots, p_{n}\right]$ gives the first $\mathrm{m}$ power functions when the first $\mathrm{n}$ are known. The cardinality is the first element of $1 \_c p u i$.

In this example card $=2$ and we search the first three power functions. We can give numerical values to the first two power functions in the list $1_{-}$cpui.

puireduc $(3,[2])$;

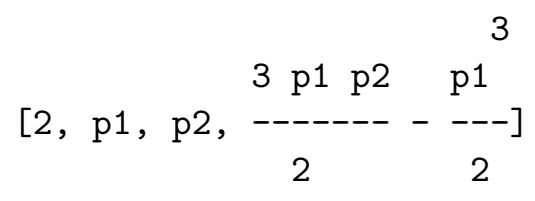

- $\operatorname{ele} 2 \operatorname{comp}\left(\mathrm{m}, 1_{-} \mathrm{cele}\right) \longrightarrow 1_{-} \mathrm{ccomp}$ gives the first $\mathrm{m}$ complete functions as functions of the elementary symmetric functions.

- $\operatorname{pui} 2 \operatorname{comp}\left(\mathrm{n}, 1_{-} \mathrm{cpui}\right) \longrightarrow 1_{-} \mathrm{ccomp}$ gives the first $\mathrm{m}$ complete functions as functions of the power functions.

- $\operatorname{comp} 2 \mathrm{ele}\left(\mathrm{n}, 1_{-} \mathrm{ccomp}\right) \longrightarrow 1_{-} \mathrm{cele}$ gives the first $\mathrm{m}$ elementary symmetric functions as functions of the complete functions.

- comp2pui (n, 1_ccomp) $\longrightarrow$ 1_cpui gives the first $m$ power functions as functions of the complete functions.

- mon2schur(liste) $\longrightarrow$ pc compute the Schur functions as functions of the monomial forms. The list appearing as argument is a $p$-uple $I$ of intergers, it represents $S_{I}$, the Schur function of index $I$ (see Section 1.2). 
- $\left.\operatorname{schur} 2 \operatorname{comp}\left(\mathrm{P},\left[\mathrm{h} i_{1}, \ldots, \mathrm{h} i_{q}\right]\right)\right) \longrightarrow$ list of lists

The polynomial $\mathrm{P}$ is $\mathcal{A}\left[h_{1}, \ldots, h_{q}\right]$, where the $h_{i}$ are the complete functions. This function expresses $\mathrm{P}$ as a function of the Schur functions, denoted by $\mathrm{S}_{I}$ in MACSYMA. It is imperative to express the complete functions by means of a letter $\mathrm{h}$ "concatenated" with an integer (ex: h2 or h5).

We first verify that the Schur function $S_{(1,1,1)}$ is equal to the third elementary symmetric function and that $S_{(3)}$ is equal to the third complete function (this is a general result).

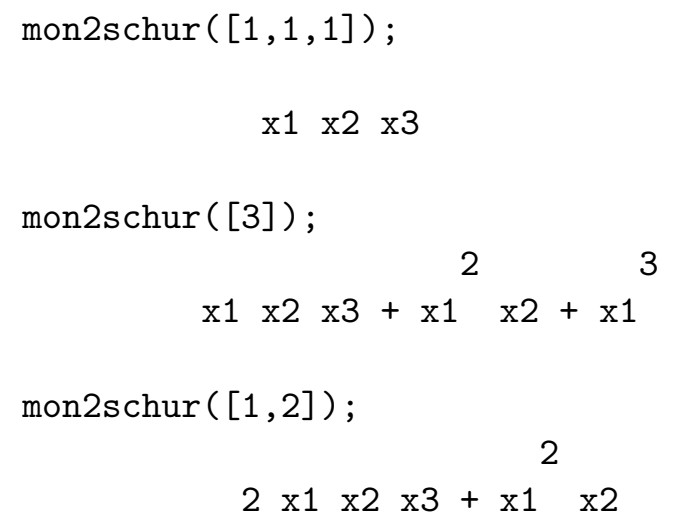

Let us see on two example, how with a circular set of operations we can go back to the initial lists $[3, \mathrm{p} 1, \mathrm{p} 2, \mathrm{p} 3]$ and $[[3, \mathrm{~h} 1, \mathrm{~h} 2, \mathrm{~h} 3]$.

a1 : $\operatorname{pui2comp}(3,[3])$;

$$
\begin{aligned}
& 23 \\
& {\left[3, \mathrm{p} 1, \begin{array}{ccccc}
\mathrm{p} 2 & \mathrm{p} 1 & \mathrm{p} 3 & \mathrm{p} 1 \mathrm{p} 2 & \mathrm{p} 1 \\
-- & +--- & -- & +---- \\
2 & 2 & 3 & 2 & 6
\end{array}\right.}
\end{aligned}
$$

a2 : comp2ele(3, a1);

$$
\left[3, \mathrm{p} 1, \begin{array}{ccccc}
\mathrm{p} 1 & \mathrm{p} 2 & \mathrm{p} 3 & \mathrm{p} 1 \mathrm{p} 2 & \mathrm{p} 1 \\
--- & -- & -- & ---- & +---
\end{array}\right]
$$

a3 : ele2pui $(3, a 2)$;

$$
[3, \mathrm{p} 1, \mathrm{p} 2, \mathrm{p} 3]
$$

a4 : comp2pui $(3,[])$;

$$
\left[3, \mathrm{~h} 1,2 \mathrm{~h} 2-\mathrm{h} 1{ }^{2}, 3 \mathrm{~h} 3-3 \mathrm{~h} 1 \mathrm{~h} 2+\mathrm{h} 1{ }^{3}\right]
$$

a5 : pui2ele $(3, \mathrm{a} 4)$;

$$
\left[3, \mathrm{~h} 1, \mathrm{~h} 1{ }^{2}-\mathrm{h} 2, \mathrm{~h} 3-2 \mathrm{~h} 1 \mathrm{~h} 2+\mathrm{h} 1{ }^{3}\right]
$$

a6 : ele2comp $(3, a 5)$;

$$
[3, \mathrm{~h} 1, \mathrm{~h} 2, \mathrm{~h} 3]
$$

In the next example we show how to express a Schur function through the monomial forms (see the label c48), and then through the complete functions (c50), the elementary symmetric functions (c51) and the power functions (en c52). 


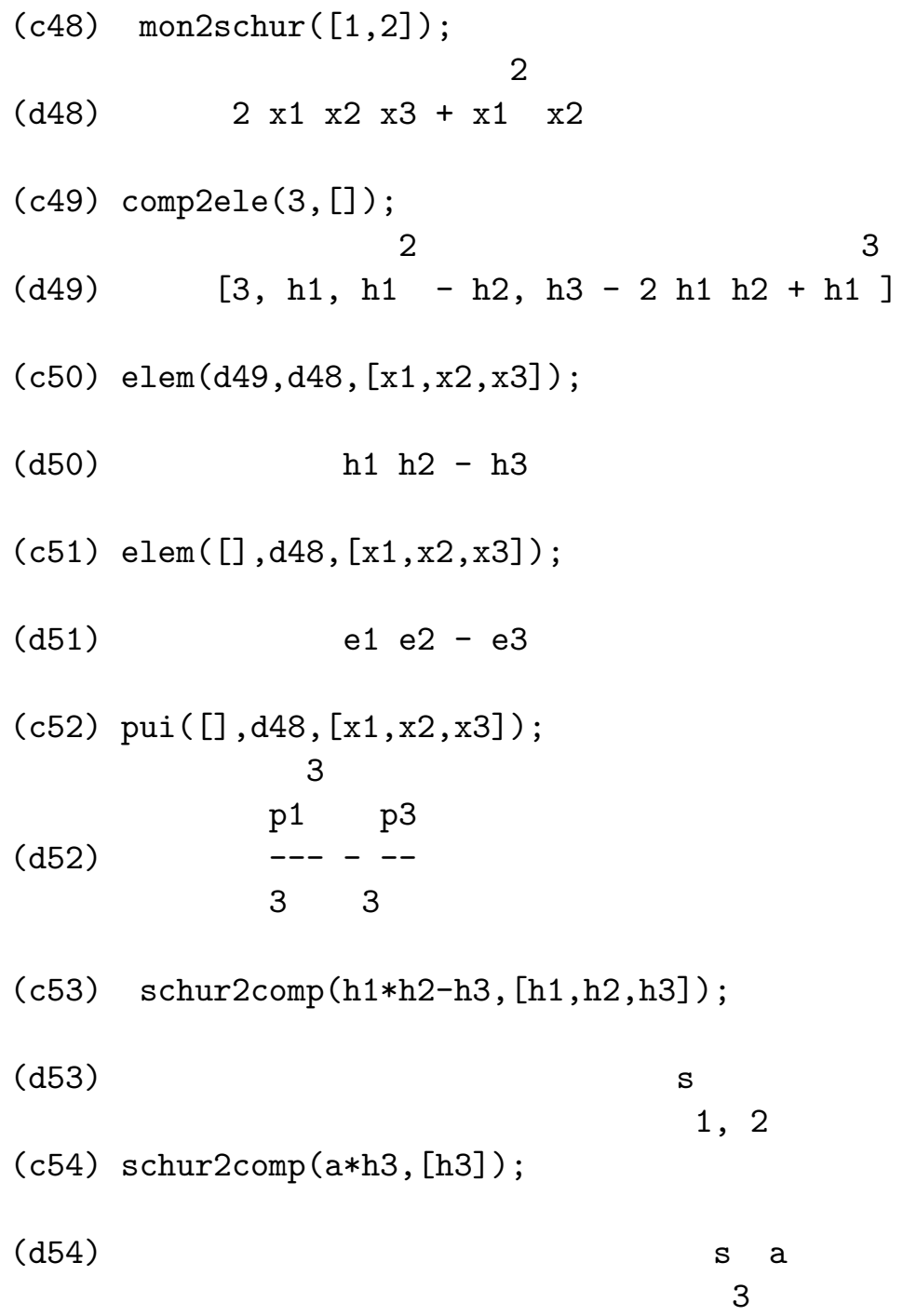

In the last instruction we have obtained the polynomials $h_{1} h_{2}-h_{3}$ and $h_{3}$ on the basis of the Schur functions.

\subsection{Direct images}

In this section, we apply the previous functions to the transformations of polynomial equations.

The direct image intruces in $[\mathrm{G}, \mathrm{L}, \mathrm{V}]$ or in $[\mathrm{V} 2]$ can represente the The resultant (function resulsym), the resolvents, such as Galois or Lagrange's (see [V2] chapter 9 p.91), or more generally minimal polynomials, can be seen as direct images ([G,L,V] and [V2]). Suppose that $\mathcal{A}$ is a field $k$. Let $f$ be a function in $R_{D}$ and let $P_{1}, P_{2}, \ldots, P_{p}, p$ polynomials of degrees $d_{1}, d_{2}, \ldots, d_{p}$, respectively. Associate with each $P_{j}$ the set $\left(a_{1}^{(j)}, \ldots, a_{d_{j}}^{(j)}\right)$ of its $d_{j}$ roots in an algebraic closure $K$ of $k$ in an arbitrary order $(1 \leq j \leq p)$, and choose an evaluation map $E_{a}: R_{D} \longrightarrow K$ which is an algebra homomorphism which sends the variable $x_{i}^{(j)}$ to $a_{i}^{(j)}$. The direct image $f_{*}(P)$ is the univariate polynomial whose roots are the images under the map $E_{a}$ of the elements of the $f$-orbite $O_{S_{D}}(f)$, i.e.:

$$
f_{*}(P)(x)=\prod_{g \in O_{S_{D}}(f)}\left(x-E_{a}(g)\right) .
$$

- $\operatorname{resulsym}(p, q, x) \longrightarrow \operatorname{resultant}(p, q, x)$

Computes the resultant of the two polynomials, $p$ and $q$, using changes of basis on sym- 
metric functions. The computation is not symmetric in $\mathrm{p}$ and $\mathrm{q}$. The computing time is best when the degree of $p$ is less than the degree of $q$.

- direct $\left(\left[P_{1}, P_{2}, \ldots, P_{p}\right], \mathrm{y}, \mathrm{f},\left[\operatorname{lvar}_{1}, \operatorname{lvar}_{2}, \ldots, \operatorname{lvar}_{p}\right]\right) \longrightarrow f_{*}\left(P_{1}, P_{2}, \ldots, P_{p}\right)(y)$ where the lists $\operatorname{lvar}_{i}$ representing $X$ (see p.3) allow us to find the type of the function $f$.

We now compute the direct image in two different ways. The first one uses, at the top level, the previous functions in order to obtain the elementary symmetric functions of the roots of the polynomial given by the function direct (which is the second way). We can change the flag direct. If it is puissances (the default value) the function direct uses the function multi_pui. If it is elementary, the function direct uses the function multi_elem (generally less efficient).

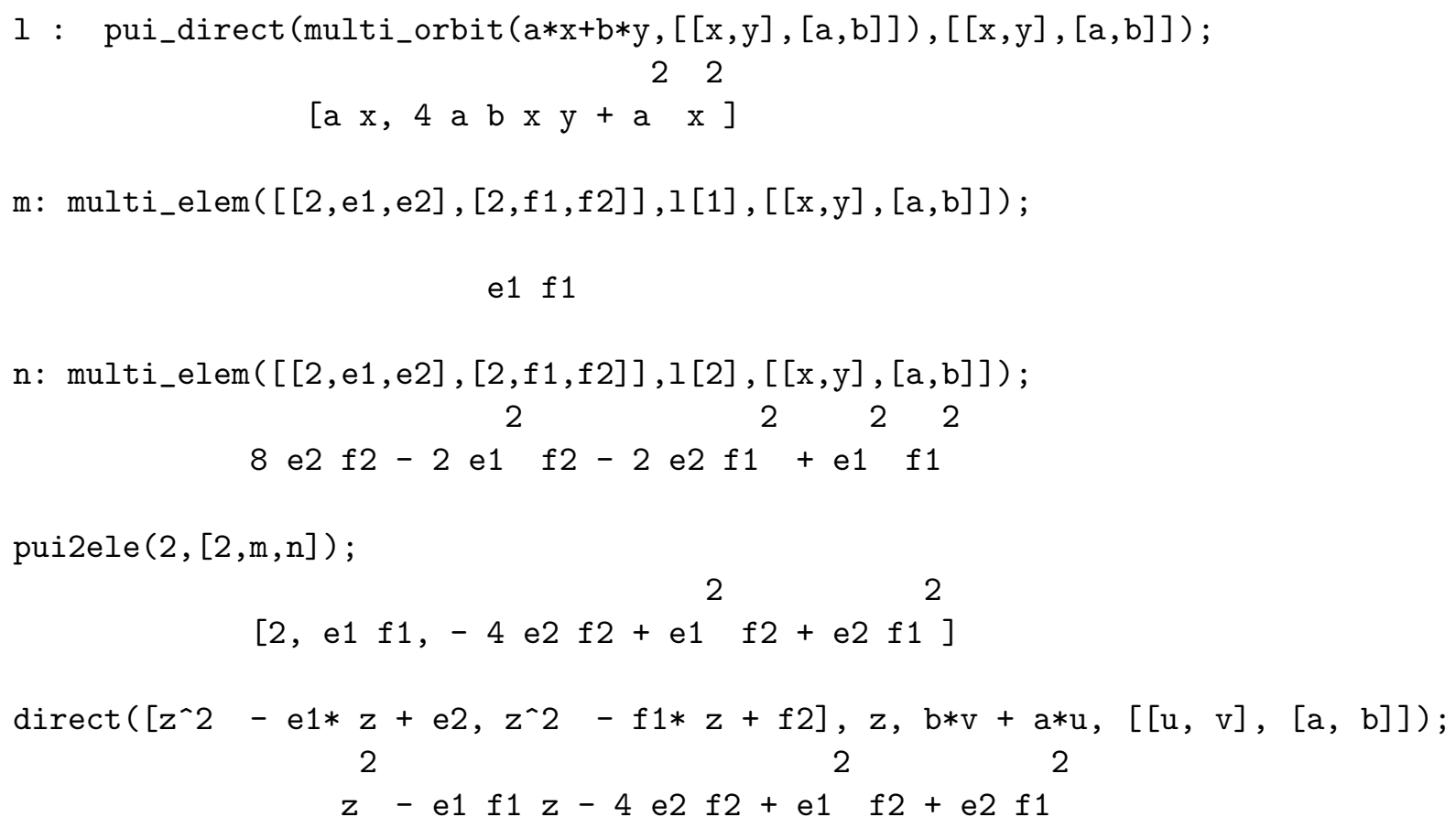

The coefficients of this polynomial in $z$ are equal (up to a sign) to the elementary symmetric functions obtained before.

- $\operatorname{somrac}\left(1_{-} e l e, k\right) \longrightarrow P(e 1, \ldots, e n)(x)$

gives the polynomial whose roots are the sums $k$ by $k$ of the roots of $p$. The polynomial $p$ is represented by the elementary symmetric functions of these roots, listed in $1_{-}$ele. Here the list 1 ele cannot be completed by formal values. If the flag somrac is pui (défault value), the function somrac uses the function pui, and if it is elem then it uses the function elem.

- prodrac $\left(I_{-} e l e, k\right)$ is the same function, but here we transform the polynomial using a product instead of the sum.

We remark that these functions are special cases of direct images.

For example, take the polynomial $x^{4}-x^{3}-25 x^{2}+25 x$ :

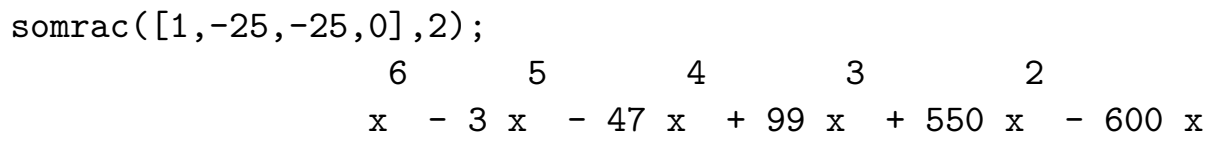




\subsection{Power function on a particular alphabet}

Let $k$ be a field.

- $\operatorname{pui}$ direct $\left(\left[f_{1}, \ldots, f_{q}\right],\left[\operatorname{lvar}_{1}, \ldots, \operatorname{lvar}_{p}\right]\right)$

Hypotheses : each $f_{i}$ is a polynomial in $k[X]$ (see the definition of $X$ in the first section), and each symmetric function on the alphabet $A=\left(f_{1}, f_{2}, \ldots, f_{q}\right)$ is multisymmetric under $S_{D}$ in $R_{D}$ (i.e. it is in $R_{D}^{S_{D}}$ ). This is the case when $f=f_{1}$, the function defined in subsection 2.6, and the alphabet represents the orbit under $S_{D}$, a product of symmetric groups. The function pui_direct computes the first $q$ power functions on the alphabet $A$. As these functions are multi-symmetric in $R_{D}$, the function pui_direct gives the power function in a contracted form in $R_{D}^{S_{D}}$ (see subsection 1.2).

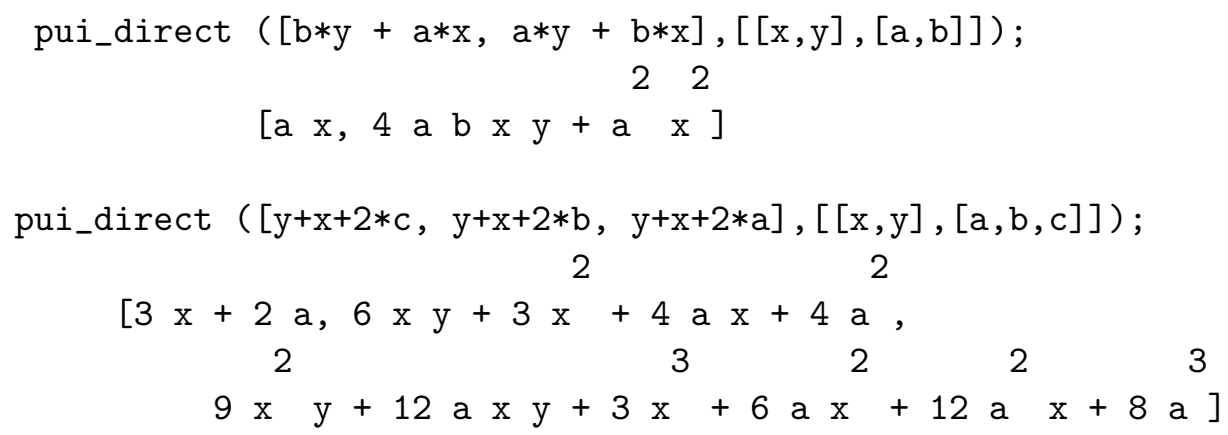

\section{References}

[Andrews] 1976, George E. Andrews, The theory of partitions, Encyclopedia of Mathematics and its Applications, Vol. 2, Section Number Theory, Addison Wesley.

[Girard], 1629, Invention Nouvelle en Algèbre, Amsterdam.

[G,L,V] 1988, M. Giusti, D. Lazard, A. Valibouze, Symmetric polynomials and elimination, Notes informelles de Calcul Formel IX, Prépublication du Centre de Mathématiques de l'Ecole Polytechnique, M810.0987.

[G,L,V] 1988, M. Giusti, D. Lazard, A. Valibouze, Algebraic transformation of polynomial equations, symmetric polynomials and elimination, Proceedings of ISSAC-88 (Roma, Italy), Springer-Verlag.

[Lascoux], Alain, 1984-1985, La résultante de deux polynômes, Séminaire d'Algèbre M.P. Malliavin.

[Lascoux, Shützenberger] 1985, A. Lascoux, M.P. Schützenberger, Formulaire raisonné de fonctions symétriques, L.I.T.P, U.E.R. MATHS, Paris 7, L.A. 248.

[Macdonald], I.G., 1979, Symmetric functions and Hall polynomials, Clarendon Press, Oxford.

[V1] 1897, A. Valibouze, Fonctions symétriques et changements de bases, Proceedings of the European Conference on Computer Algebra EUROCAL '87 (Leipzig, RDA), Springer-Verlag.

[V2] 1988, A. Valibouze, Manipulations de fonctions symétriques, Thèse de l'Université Paris VI. 